\title{
Charge transport in organic semiconductors: From incoherent to coherent
}

\author{
YAO Yao, SI Wei, YANG WenChao \& WU Chang-Qin* \\ State Key Laboratory of Surface Physics, Department of Physics, Fudan University, Shanghai 200433, China
}

Received January 30, 2013; accepted April 22, 2013; published online June 21, 2013

\begin{abstract}
Quantum decoherence in organic charge transport is a complicated but crucial topic. In this paper, several theoretical approaches corresponding to it, from incoherent to coherent, are comprehensively reviewed. We mainly focus on the physical insight provided by each theory and extent of its validity. The aim of this review is to clarify some contentious issues and elaborate on the promising perspectives provided by different approaches. The device model approaches based on both continuous and discretized treatments of the transporting layer will be first discussed. The prominent focus of this review will be devoted to the dynamic disorder model and its variants considering that it is the most promising approach to tackle charge transport problems in organic materials. We will also address other theories such as the variational method.
\end{abstract}

organic semiconductors, charge transport, decoherence

Citation: Yao Y, Si W, Yang W C, et al. Charge transport in organic semiconductors: From incoherent to coherent. Chin Sci Bull, 2013, 58: 2669-2676, doi: $10.1007 /$ s11434-013-5931-y

As in all other molecular systems, the electronic coherence is an important aspect in the transport in organic semiconductors (OSCs). The phonon spectrum, consisting of both the intra- and inter-molecular ones, covers an extensive regime and makes almost all the theories about coherence inefficient. As opposed to the biomolecular systems in which the regime of interest is where the long-lived coherence exists and is thus typically specific [1], a very wide range of issues for organic semiconductors spanning completely incoherent (classical) to completely coherent (quantum) exist [2,3]. For example, to study the mobility in a given molecular system, one can utilize classical (device model [4] or kinetic Monte Carlo simulation [5]), semiclassical (surface hopping algorithm [6]), mixed quantum classical (dynamic disorder model [7] and Ehrenfest method [8]), and quantum (variational ansatz) theories. The diverse theoretical treatments make the problems obscure for experimentalists and theoreticians alike. In this review, we will briefly introduce each of these approaches and attempt

*Corresponding author (email: cqw@fudan.edu.cn) to provide insights related to their applicability.

The charge carriers in OSCs are recognized to be (positively or negatively) charged polarons, due to the strong self-trapping effect from lattice distortion and vibration. Polarons could be small or large, depending on the relative scales of intra- and inter-molecular interactions [9]. In a lattice model, these interactions could be described by diagonal and off-diagonal electron-phonon couplings. A typical single-carrier Hamiltonian then should be as follows [10]

$$
\hat{H}=\sum_{i j} \varepsilon_{i j} \hat{c}_{i}^{\dagger} \hat{c}_{j}+\sum_{k} \hbar \omega_{k} \hat{b}_{k}^{\dagger} \hat{b}_{k}+\sum_{i j k} \hbar \omega_{k} \alpha_{i j k}\left(\hat{b}_{k}^{\dagger}+\hat{b}_{k}\right) \hat{c}_{i}^{\dagger} \hat{c}_{j},
$$

here $\varepsilon_{i j}$ is the on-site energy for $i=j$ and the transfer integral for $i \neq j ; \hat{c}_{i}^{\dagger}\left(\hat{c}_{i}\right)$ is the creation (annihilation) operator of a carrier on site $i ; \omega_{k}$ is the frequency of the $k$-th phonon mode; $\hat{b}_{k}^{\dagger}\left(\hat{b}_{k}\right)$ is the creation (annihilation) operator of the phonon of the $k$-th mode; $\alpha_{i j k}$ is the electron-phonon coupling constant. In some polymers, the charge carriers might be some mobile charged defects or 
radicals [11]. The role of this type of carriers is to increase the intrinsic concentration of free carriers and also the dielectric constant. It is deemed to contribute mostly to the high conductivity of these polymers, but a comprehensive picture for it is still lacking.

A commonly encountered problem in OSCs is to account for disorder. Impurities, molecular mismatches, entangled kinks, segment and phase edges may all contribute to the static disorder [12], whereas the molecular vibrations and displacements make up the dynamic disorder [7]. Normally, in the presence of only static disorder, the carriers must be completely localized to a very small region and become immobile [5]. Two mechanisms may enable the carrier to escape. Firstly, if the density of carriers increases dramatically, all the traps formed by static disorder will be filled and the additional carriers are then movable [13]. Secondly, dynamic disorder could help break the localization and recover the coherence, eventually making the carriers hop out of the deep potential well. The former case occurs in the organic field effect transistors (OFETs), the space charge limited organic light emitting diodes (OLEDs), and the organic bulk heterojunction solar cells. The latter case exists in more general circumstances, especially in organic crystals and polymers.

Regardless of the detailed mechanisms, the origin and the physical picture of decoherence are of crucial importance in OSCs. Generally speaking, decoherence originates from environment induced superselection [14]. However, little is known about the environmental effects in organics. In this context, a few experimental results could be referred to for the estimation of, for example, the typical decoherence time in these materials. The first-principle calculations can provide the distribution of vibrational frequencies and coupling strengths, but cannot describe how these results are related to the decoherence [15]. Without this basic knowledge, it is difficult to distinguish the different regimes from the entangled experimental measurements. For example, the temperature dependence of mobility in most OSCs shows quite strange nonmonotonic behavior [16], in which incoherent hopping and coherent bandlike behaviors coexist with each other. No characteristic value of temperature or time is clarified. The existing estimations are from some non-transparent assumptions, such as the results of variational theory. More careful research along this aspect is thus warranted.

The optoelectronic and electro-optic processes are of central importance in the study of OSCs, which show promising potential as future energy sources and display devices. The carrier injection and recombination at the interfaces are very important processes and many aligned issues are still under active debate. On the transport aspect, one would be interested in the role of scattering processes. Especially, processes in the hopping regime, such as the polaron-exciton quenching, triplet-triplet annihilation, site/ spin blocking, are discussed frequently [17]. While moving in the transporting layer, the carrier may get localized in some coherent region (e.g., a single molecule in amorphous small molecule materials) until next hopping takes place. The waiting time is of the order of magnitude of $1 \mathrm{~ns}$, within which the carrier has a sufficiently long time to interact with other particles. This interaction induces local entanglement, which could then influence the hopping rate [18]. Detailed analysis should be rather complicated but important, considering the rich and novel physics these processes contain.

The paper is organized as follows. The next section is contributed to the introduction of device model. The rate equation algorithm and kinetic Monte Carlo simulation is reviewed in the third section, where the Einstein's relation is also elaborated upon. The dynamical disorder model and relative theories for it are discussed in the fourth section, which is followed by discussion on the other approaches, such as the variational theory. Conclusions are drawn in the final section.

\section{Device model}

In most cases, the organic semiconducting devices based on both small molecules and polymers function in the incoherent hopping regime (e.g. room temperature, modest electric field and disorder, and low carrier concentration), and the approaches on the macroscopic, phenomenological, and (semi-) classical level should be sufficiently applicable and efficient to simulate the device measurements. In this section, we first give a brief description of the device model. The notion "device model" could be ambiguous considering its occurrence in nearly every theory. Nevertheless, two types of methods for modeling the device are most frequently used: One is based on drift-diffusion equations, and the other is the rate (master) equations. In this section, we will focus on the former one, and the latter will be postponed to the next section. Hereafter, the device model will be used to refer in the context of drift-diffusion equations.

The basic idea of the device model is to assume the organic transporting layer to be a continuous medium. The drift-diffusion equations that account for two types of carriers, electrons and holes, when coupled with the Poisson's equation $[4,19]$, yield:

$$
\begin{gathered}
\frac{\partial p}{\partial t}=-\frac{1}{e} \frac{\partial J_{p}}{\partial x}+k_{\mathrm{d}} N-R, \\
\frac{\partial n}{\partial t}=\frac{1}{e} \frac{\partial J_{n}}{\partial x}+k_{\mathrm{d}} N-R, \\
\frac{\partial E}{\partial x}=\frac{e}{\varepsilon_{0} \varepsilon}(p-n),
\end{gathered}
$$

where $p(n)$ is the hole (electron) density, with $J_{p}\left(J_{n}\right)$ the corresponding electric current from holes (electrons); $k_{\mathrm{d}}$ is a constant dissociation rate for the charge transfer excitons (or polaron pairs) from the Onsager-Braun model with $N$ the 
exciton concentration, which could either be set as an exponential function with distance from the site of absorption of light, or given by solving the diffusion equation of excitons; $R$ is the recombination rate; $E$ is the electric field; $e$ is the electron charge; $\varepsilon_{0}$ and $\varepsilon$ are the static and relative dielectric constants, respectively. The hole/electron current $J_{p / n}$ are of the drift-diffusion form:

$$
\begin{aligned}
& J_{p}(x)=e \mu_{p}\left(p E-\frac{k_{B} T}{e} \frac{\partial p}{\partial x}\right), \\
& J_{n}(x)=e \mu_{n}\left(n E+\frac{k_{B} T}{e} \frac{\partial n}{\partial x}\right),
\end{aligned}
$$

where $\mu_{p}\left(\mu_{n}\right)$ is the mobility of holes (electrons). The Einstein's relation $D_{p / n}=\mu_{p / n} k_{B} T / e$ has been employed for the diffusion current. In these equations, the mobility is treated as a phenomenological parameter. In some cases, a field dependent mobility is applied caused by the disordered conducting paths. However, the weakness of the continuous medium assumption is the lack of the appropriate treatment of the disorder.

Following the straightforward idea, the most important step is to choose the appropriate recombination and injection models. There are three typical recombination models. The most commonly used among them is the Langevin bimolecular recombination model [20], in which the recombination rate is expressed as:

$$
R=\gamma\left(n p-p_{e} n_{e}\right),
$$

where $p_{e}\left(n_{e}\right)$ is the hole (eletron) densities in the equilibrium state and the coefficient $\gamma$ is given by

$$
\gamma=e\left(\mu_{n}+\mu_{p}\right) / \varepsilon_{0} \varepsilon
$$

In organic bulk heterojunction solar cells, the bimolecular recombination usually occurs at the donor/acceptor interface, so the recombination rate is limited by the charge carriers with smaller mobility. The second recombination model takes into account this effect and the coefficient $\gamma$ is modified as [21]:

$$
\gamma=\frac{e}{\varepsilon_{0} \varepsilon} \min \left(\mu_{n}, \mu_{p}\right)
$$

The third model accounts for the monomolecular recombination due to the charge carrier trapping by the impurities or defects in the active layer. According to the ShockleyRead-Hall (SRH) theory [22,23], the recombination rate can be written as:

$$
R_{\mathrm{SRH}}=\frac{C_{n} C_{p} N_{t}\left(n p-n_{e} p_{e}\right)}{C_{n}\left(n+n_{e}\right)+C_{p}\left(p+p_{e}\right)},
$$

where $N_{t}$ is the density of traps; $C_{p}\left(C_{n}\right)$ is the capture coefficients of holes (electrons).
Charge carriers are injected into the organic layer at the metal/organic contact. The injection current usually contains the thermionic injection current and the field-induced tunneling current [4]. In most cases when the internal field is not extremely high, the latter current can be safely neglected. For example, the thermionic injection current for holes at position $x=0$ is given as:

$$
J_{\text {th }}=A T^{2} \exp \left(-\phi_{p} / k_{B} T\right)
$$

where $A$ is the Richardson's constant, and $\phi_{p}$ is the injection barrier of holes. If the electric field is in the proper direction, the decrease in potential due to the image charge effect should be taken into account as

$$
\phi_{p}=\Phi_{p}-e \sqrt{e|E(0)| / 4 \pi \varepsilon_{0} \varepsilon}
$$

where $E(0)$ is the electric field at $x=0$. There also exists a reversing recombination current $J_{\text {ir }}$ formed by the carriers flowing back to the electrode. In the equilibrium state, $J_{\text {th }}$ and $J_{\text {ir }}$ must satisfy the detailed balance condition and thus cancel each other. As a result $J_{\text {ir }}$ is of the form

$$
J_{\mathrm{ir}}=A T^{2} \cdot \frac{p}{p_{0}},
$$

for holes, with $p_{0}$ the intrinsic density of hole. For electrons, the above equations take similar forms. By combining $J_{\text {th }}$ and $J_{\text {ir }}$ the interfacial current is obtained:

$$
J=J_{\text {th }}-J_{\text {ir }},
$$

which gives the boundary condition for the continuity eqs. (2) and (3) for charge carriers. Now the equations of the device model can be solved numerically and all the relevant macroscopic quantities such as current, carrier density and internal electric field can be obtained straightforwardly.

By employing the device model method, we have investigated some dynamical and steady state electronic processes in organic solar cells. In order to explain the experimental finding of the polarity change of transient photovoltage curves in single-layer organic solar cells shortly after the laser illumination is turned on, we proposed the important role of the interfacial dissociation of photo-generated excitons in producing free charge carriers [24]. The time evolution of the transient photocurrent signal was simulated, and the results are consistent with experimental findings [25]. The surface loss effect due to the carrier extraction from the wrong electrode was examined, and we found the surface losses are enhanced with increased interfacial dissociation rate or diffusivity of the charge transfer excitons [26]. In a recent work [27], we investigated a particular type of cell degradation effects which are reflected by the $S$-shaped deformation of $J$ - $V$ curves.

So far, the device model has proved to be the most accurate method for rebuilding the device measurements. The 
typical diode-like $J$ - $V$ curves, S-shaped $J$ - $V$ curves, open circuit voltage/short circuit current/filling factor, and (timeinvolved) photocurrent and voltage, can all be simulated within the same theoretical framework. To a large extent, the behavior of OSCs is similar to that of the traditional semiconductors. However, what distinguishes OSCs is the presence of the strong disorder induced by the soft phonon modes. This factor gives rise to the temperature-field- and structure-dependence of mobility. Considering that the performance and efficiency of the devices based on OSCs is lower than those based on traditional semiconductors, many peculiar properties under extreme device operational conditions are not crucial at least at the current level of technology. With further sophistication in technological developments, however, the device model may be rendered insufficient to obtain correct results.

\section{Rate equation and Kinetic Monte Carlo simulation}

An intuitive way to consider the influence of disorder is to utilize the rate equation [28]. Different from the assumption of continuous medium of the device model, here the transporting layer is discretized into a number of sites, with each site representing a single molecule, a conjugated segment in polymers, a percolating path, or a coherent region. The carrier is supposed to incoherently hop among these sites with some specific form of hopping rate. The most commonly employed form of rate equation is:

$$
\frac{\mathrm{d} p_{i}}{\mathrm{~d} t}=\sum_{j}\left[v_{i j} p_{i}\left(1-p_{j}\right)-v_{i j} p_{j}\left(1-p_{i}\right)\right],
$$

where $v_{i j}$ is the rate of the carrier to hop from site $i$ to $j$; $p_{i}$ is the probability that site $i$ is occupied by a carrier. The initial state of carrier distribution must be specified and its choice depends on the specific experimental conditions. The equations are then solved self-consistently and the mobility can be calculated as

$$
\mu=\frac{\sum_{i j} v_{i j} p_{i}\left(1-p_{j}\right) r_{i j, E}}{n L^{3} E},
$$

where $r_{i j, E}$ is the distance between site $i$ and $j$ along the direction of electric field $E ; n$ is the carrier density; $L$ is the size of lattice considered.

Disorder in both the site energies and hopping constants (intermolecular distance) could be taken into account in the same framework. To this end, one can use some wellknown theories for the hopping rate, such as the MillerAbrahams theory [29], and the Marcus theory [30]. For example, the Miller-Abrahams formula reads, for the hopping rate $v_{i j}$ from site $i$ to $j$,

$$
v_{i j}=\left\{\begin{array}{ll}
\left.v_{0} \exp \left(-r_{i j} / r_{0}\right) \exp \left[-\left(\varepsilon_{j}-\varepsilon_{i}\right) / k_{B} T\right]\right), & \varepsilon_{j}>\varepsilon_{i} \\
v_{0} \exp \left(-r_{i j} / r_{0}\right), & \varepsilon_{j} \leqslant \varepsilon_{i}
\end{array},\right.
$$

where $v_{0}$ is the attempt-to-escape frequency; $r_{i j}$ is the distance between site $i$ and site $j ; r_{0}$ is the localization length; $\varepsilon_{i}$ is the electronic energy of carrier on site $i$; $T$ is temperature. Here disorder is introduced in both the energy and distance terms, by using appropriate probability distributions. One of the most famous distribution is Bässler's Gaussian disorder model (GDM) [5], in which the electronic energies of sites are drawn from the Gaussian distribution $\left(2 \pi \sigma^{2}\right)^{-1 / 2} \exp \left(-\varepsilon^{2} / 2 \sigma^{2}\right)$, where $\sigma$ is the width of the disorder distribution. One can easily find, by using GDM and solving the rate equation for one type of carrier, the diffusion coefficient behaves as $\sim \exp \left(-1 / T^{2}\right)$. While this is true for some OSCs, it is not applicable in general.

It is worth noting that, the Einstein's relation is always violated in OSCs [31]. The strong disorder makes the system far away from the thermal equilibrium, while the Einstein's relation is valid only near the equilibrium. One way to avoid this contradiction is to consider the details of local density of states, which however is not an efficient approach. Unsurprisingly then, this issue is still subject to intense research.

Typically the rate equation is not easy to solve, and the kinetic Monte Carlo (KMC) simulation poses a possible way for its solution [5]. This method originates from the simulation of the formation of metallic tips in STM. Instead of calculating the evolution of charge density, the basic idea of $\mathrm{KMC}$ is to treat the carriers one by one, namely, to first target each carrier's trajectory and to calculate the averaged property. In detail, in a simple cubic lattice with only the nearest-neighbor hopping allowed, for each instance of hopping, the carrier residing on a given site has six target sites. The hopping rate to each site follows the MillerAbrahams formula. One then generates two random numbers $\xi_{1}$ and $\xi_{2}$ uniformly distributed within [0,1], and make the hopping from site $m$ to site $\kappa$ happen if [32]

$$
\left(\sum_{n=1}^{m-1} \sum_{\alpha=1}^{6} v_{n, \alpha}+\sum_{\alpha=1}^{\kappa-1} v_{m, \alpha}\right)<\xi_{2} v_{\text {tot }}<\left(\sum_{n=1}^{m-1} \sum_{\alpha=1}^{6} v_{n, \alpha}+\sum_{\alpha=1}^{\kappa} v_{m, \alpha}\right)
$$

where $1 \leqslant m \leqslant N, 1 \leqslant \kappa \leqslant 6$. Herein, $v_{\text {tot }}$ is the hopping rate of the carrier

$$
v_{\mathrm{tot}}=\sum_{n, \alpha} v_{n, \alpha}, \tau=-\ln \left(\xi_{1}\right) / v_{\mathrm{tot}}
$$

with $\tau$ the waiting time that we want to calculate. After averaging on a number of samplings, the obtained diffusion coefficient should be similar with the analytical results. By this method, some of the present authors have investigated the field and disorder dependent mobility in the OFET, and the details of the KMC simulation were also carefully studied [32]. Meng et al. [33] have also apply this method to polymer-blend solar cell with Poisson equation coupled. However, it is necessary to emphasize that, the KMC results 
are always dominated by some deep traps in the system, since the waiting time there is extremely long and thereby bearing significance in the final results. This implies that for good conducting systems, KMC may lead to incorrect results.

\section{Mixed coherent and incoherent theories}

The approaches we described so far are predominantly applicable for the amorphous organic materials. However, a great research interest has emerged in the study of transport properties in crystalline and semi-crystalline materials [34]. These studies are motivated by the development of organic field-effect transistors based on materials such as pentacene. In such materials over a certain temperature range, the mobility is found to decrease with increasing temperature [35]. However, spectroscopic experiments have indicated that the localization length of the charge carrier spans approximately 10 molecules [36,37]. The contrast between the localized charge carriers and "band-like" temperature dependence thus poses a stiff challenge in the construction of the theoretical approaches.

\subsection{Dynamic disorder model}

To tackle the transport problem in crystalline organic materials, Troisi et al. [7] proposed the dynamic disorder model. In their study, the molecules are abstracted as transport sites on a one-dimensional chain, with the intermolecular transfer integral modulated by the lattice vibrations. For the case of one-electron, the Hamiltonian can be written as $\hat{H}=$ $\hat{H}_{\mathrm{el}}+\hat{H}_{\mathrm{la}}$, where the electronic part is

$$
\hat{H}_{\mathrm{el}}=\sum_{j} J\left[-1+\alpha\left(u_{j+1}-u_{j}\right)\right]\left(\hat{c}_{j}^{\dagger} \hat{c}_{j+1}+\hat{c}_{j+1}^{\dagger} \hat{c}_{j}\right),
$$

and the lattice part is

$$
\hat{H}_{\mathrm{la}}=\sum_{j}\left[\frac{1}{2} m \dot{u}_{j}^{2}+\frac{1}{2} m \omega_{0} u_{j}^{2}\right],
$$

here $j$ is the transfer integral; $\alpha$ is the electron-phonon coupling constant; $u_{j}$ is the displacement of the $j$-th transport site; $\hat{c}_{j}^{\dagger}\left(\hat{c}_{j}\right)$ is the creation (annihilation) operator of an electron on site $j ; m$ is the mass of the molecules and $\omega_{0}$ is the phonon frequency. The initial positions and velocities of the transport sites are randomly drawn from the distribution at temperature $T$. The initial states of electrons are chosen from the instantaneous eigenstates of the Hamiltonian according to the Boltzmann distribution. The dynamics of the electron follows the Schrödinger equation. For the dynamics of the lattice sites, the Hellman-Feynman force is added as

$$
m \ddot{u}_{j}(t)=-m \omega_{0}^{2} u_{j}(t)-\frac{\partial}{\partial u_{j}}\left\langle\psi(t)\left|\hat{H}_{\mathrm{el}}\right| \psi(t)\right\rangle .
$$

This model can be simplified further by choosing appropriate system of units. This procedure results in only two dimensionless parameters termed as the electron-phonon coupling constant $\lambda=\left(\alpha^{2} J\right) /\left(2 m \omega_{0}^{2}\right)$ and the phonon frequency $\omega=\hbar \omega_{0} / J$. If the dynamics of the lattice sites are ignored, i.e., $u_{j}$ are fixed, the electron wavefunction will remain localized. However, when it is taken into account, the electron wavefunction will spread over time, which is analogous to its diffusion. In this sense, this model is termed as the dynamic disorder model. It turns out that the variance of the wavefunction is roughly proportional to time. This makes it possible to extract a diffusion constant from the calculation, from which the mobility can be calculated by the Einstein's relation. The mobility obtained by this method exhibits a monotonic decrease with an increase in temperature over a wide range of parameters. Various other approaches for the generalization of this method have also been proposed. For example, motivated by the development in organic field-effect devices, two-dimensional models have been developed to accurately predict mobility in a certain class of materials [38]. Li et al. [39] studied the influence of the symmetric electron-phonon coupling on the transport properties, as compared to the more commonly employed anti-symmetric form.

Here we point out that, there remain several unsolved issues with the treatment proposed by Troisi et al. [31]. Of foremost importance among them is the lack of clarity on the validity of the Einstein's relation. Furthermore, the procedure to calculate mobility from diffusion constant warrants a careful examination. A more important problem concerns the coherence of the evolution of wavefunction. Although the mixed quantum-classical Ehrenfest method is used, the evolution of the wavefunction acquires a coherent form. It will keep spreading with time as a pure state. In this sense, the "localization length" keeps growing and the carrier localization is broken. This is in contradiction with either the localization length calculated from the spontaneous energy eigenstates, or that measured in experiments. This problem becomes far clearer if an electric field is added to the electronic Hamiltonian. The wavefunction of the carrier does not exhibit the expected drift motion with the electric field. This might be due to the Bloch oscillations, which is a signature of the quantum coherence due to the lack of necessary scattering mechanisms. To overcome this problem, Böhlin et al. [40] has proposed to add a dissipative term in the equation of motion of transport sites. The drift motion in the electric field is successfully recovered, but the relation between the drift mobility and the diffusion constant remains to be examined.

\subsection{Other theoretical treatments}

Following the work of Troisi, Ciuchi et al. [8, 41] studied a similar system from the perspective of correlation functions 
and the Kubo formula. The quantity they have calculated is the quantum-mechanical spread $\Delta X^{2}(t)=\left\langle[\hat{X}(t)-\hat{X}(0)]^{2}\right\rangle$, where $\hat{X}(t)$ is the position operator in the Heisenberg representation at time $t$. The derivative of this quantity is shown to be proportional to the integral of the autocorrelation function of velocity $C(t)=\langle\hat{V}(t) \hat{V}(0)+\hat{V}(0) \hat{V}(t)\rangle$, which is

$$
\frac{\mathrm{d} \Delta X^{2}(t)}{\mathrm{d} t}=2 D(t)=\int_{0}^{t} C\left(t^{\prime}\right) \mathrm{d} t^{\prime}
$$

With this relation and the Kubo formula, the optical conductivity $\sigma(\omega)$, which can be measured in experiments, can be calculated from $\Delta X^{2}$ by

$$
\sigma(\omega)=-\frac{e^{2} \omega^{2}}{v} \frac{\tanh (\beta \omega / 2)}{\hbar \omega} \operatorname{Re} \int_{0}^{\infty} \mathrm{e}^{i \omega t} \Delta X^{2}(t) \mathrm{d} t,
$$

where $\beta=1 / k_{B} T$. In another perspective, the quantity $D(t)$ can be seen as a quantum diffusivity, which corresponds to the diffusion constant in Troisi's work. They found that if the dynamic disorder is incorporated, the diffusivity will gradually increase with time. However, if the dynamic disorder is frozen, the diffusivity will tend towards zero during a sufficiently long time, which is also not realistic. To get a constant diffusivity at long times, which is what the experiments yield, they introduced the relaxation time approximation and the actual diffusivity can then be given as $D_{\mathrm{RTA}}(t)=D(t) \exp (-t / \tau)$, where $\tau$ is the relaxation time. The results obtained with this approach are in excellent agreement with the experimental findings [42]. Apart from the above approach based on the model Hamiltonian, the effect of dynamic disorder on transport properties can also be studied from carrier hopping point of view. Geng et al. [43-45] pointed out that the initial assumptions for the derivation of the Marcus formula of carrier hopping rate, i.e., low frequency and high temperature is not suitable for the case of organic semiconductors. The effect of high-frequency intramolecular phonon modes has to be taken into consideration. They combined the density functional theory calculations and the molecular dynamics simulations to directly calculate the charge transfer rate beyond the above approximations, which is named as the tunneling enabled hopping model. The dynamic disorder effect is included by the change in the intermolecular transfer integral obtained from the molecular dynamics. The resulting rate is incorporated in the kinetic Monte Carlo simulations to simulate the diffusion process. As before, the mobility is calculated from the Einstein's relation. It was found that the mobility decreases with an increase in temperature, thereby contrasting with the results from the Marcus formula. The calculated mobility is however in quantitative agreement with the experimental value. Another interesting finding is that the dynamic disorder could enhance the mobility at high temperature, although opposite effect is observed at low temperature. In this way, a mechanism for transport via localized electronic states with "band-like" temperature dependence is claimed. However, it should be noted that the actual role of dynamic disorder in the charge transport of organic materials is still not clear. For example, by different treatments, the calculated mobility can be either sensitive [7] or insensitive [44] to the dynamic disorder. While some preliminary experimental results appear to not be favorable towards the effect of dynamic disorder [46], further theoretical and experimental studies are certainly needed to clarify its role.

\subsection{Measurement-induced incoherent hopping}

The inadequacies in the aforementioned approaches warrants for the introduction of new physics into the study of transport with the dynamic disorder model. As an important example of the decoherence effect, it has potential to overcome the breakdown of carrier localization, and to be able to describe the transition between the coherent and incoherent transport $[47,48]$. Decoherence can be brought about by the scattering effects from high-frequency intramolecular phonons [49]. It has similar effects to a quantum system as the iterative quantum measurement, which has been one of the effective ways to get the localized carriers moving in low dimensional disordered Anderson models [49].

Recently, we proposed a physical picture of decoherence effect in dynamic disorder model, by incorporating a decoherence time $t_{\mathrm{d}}$ [50]. In this approach, the wavefunction of a single carrier starts from a single site at time $t=0$. It evolves with time until time $t=t_{\mathrm{d}}$, when the decoherence effects collapse the wavefunction of the carrier to a single site again, namely measure the carrier in a quantum manner. The site that the wavefunction collapses to is chosen according to the probability distribution from the wavefunction at $t=t_{\mathrm{d}}$. This physical picture can be termed as the frequent projection method. From the point of view of statistical ensembles, it could be described by the equation of motion of the density matrix $\rho$, by adding a decay term for the off-diagonal matrix elements. The nonvanishing offdiagonal matrix element is a signature of the quantum coherence between different sites. If $t_{\mathrm{d}}$ is small, the nondiagonal elements remain small in the evolution, and thereby the quantum coherence is suppressed.

The results of these calculations, when employing the same parameters as those used by Troisi, show that the diffusion of the carrier wavefunction slows down due to the addition of $t_{\mathrm{d}}$. As $t_{\mathrm{d}}$ increases, the diffusion constant gradually increases to the value of Troisi's work. The temperature dependence of diffusion constants is also investigated and found to change from negative to positive influence as the intermolecular transfer integral $J$ decreases. This could be seen as an indication of the transition from coherent to incoherent transport. For intermediate values of transfer 
integral, the dependence is not monotonous and exhibits more complicated behavior which calls for further study. Furthermore, the spatial extent of the carrier wavefunction is calculated in this framework and found similar to the carrier localization length determined by experiments when the decoherence time is $1 \mathrm{ps}$, which serves as an estimation to the decoherence time in real situations. Further work may include the realization of this phenomenological physical picture from more detailed models of electron-phonon interactions, such as the Holstein model. It is also meaningful to explore the relation of this method with other approaches to introduce scattering into the dynamic disorder model, such as the relaxation time approximation mentioned above.

\section{Semiclassical and variational approaches}

In this section we comment on some other theoretical methods which are relevant to the charge transport in OSCs. They are all based on the Holstein-like models, and take into account the electron-phonon interactions. This should be more a priori idea to study the coherent and incoherent transitions. The Holstein model has no exact solutions for the whole parameter regime. More importantly, the transition point between coherent and incoherent is within the parameter region that is the hardest to deal with. Therefore, different theoretical approaches join in to make contributions. Till now, the development of methods is the core topic in this subfield.

The idea of semiclassical simulations relies on the mapping of electronic Hamiltonian from quantum to classical domain, by using a path integral formulism and a frozen Gaussian wavepacket [51]. The central point of this method is to appropriately deal with the quantum fluctuation without a growth in the computational cost. The most famous way to achieve this is the so-called initial value representation [52], whose idea is to express the time-involved position and momentum via their initial values. This method should be quite efficient, but the numerical precision is hard to control. Another way is the surface hopping algorithm, which treats the trajectory of phonons separately and makes the hopping eventually happen by a specific criterion. Interested readers are recommended to read Tully's review paper on this issue [6].

Alternatively, one may try to solve the model on some approximating level. The variational theory is mostly used. The pioneer work is by Yarkony and Silbey [2], who studied very carefully the coherent and incoherent components of the mobility. The variational parameter is mainly the displacement of phonon, as the phonons are supposed to be in the coherent states

$$
|D\rangle=\sum_{n} A_{n}|n\rangle \otimes \sum_{l}\left(B_{l} b_{l}^{\dagger}-B_{l}^{*} b_{l}\right)|0\rangle_{p h},
$$

here, $A_{n}$ and $B_{l}$ are the variational parameter for electron on $n$-th site and phonon with $l$-th mode, respectively. One can then calculate the total energy and minimize it to obtain the ground state of the system. In different theories, the trial states can be slightly different, but the physical motivations behind them are identical. Within this theoretical framework, both weak and strong electron-phonon couplings could be described, but in the intermediate case, which is the most important, it loses the efficiency.

\section{Concluding remarks}

OSCs have emerged as promising materials for their use in a number of advanced applications. However, so far their commercial potential has not been developed sufficiently relative to the huge variety and low cost of organic molecules. In this field, the experiment and theory were usually going on different branches. The key point is, in our opinion, the materials, technologies and observed phenomena are too diverse. The theoretical approaches have no common point as well, as we have indicated above. Even so, we still think the electronic coherence is in the core position. Actually, the electronic coherence in charge transport in OSCs has been studied extensively in the literature. One can make a large effort on this issue on the present stage. No matter which method mentioned above, it can definitely contribute to the community. For example, the clarification of the unknown decoherence time scale could help the theoretician to distinguish coherent and incoherent regime more clearly. More novel physics could be discovered, if the fundamental problems were solved. Therefore, one would expect more comprehensive works on the charge transport in the near future.

This work was supported by the National Natural Science Foundation of China (11134002, 10874028), and the National Basic Research Program of China (2009CB929204, 2012CB921402). The authors thank Dr. Prathamesh Shenai for reading the manuscript.

1 Ishizaki A, Fleming G R. Theoretical examination of quantum coherence in a photosynthetic system at physiological temperature. Proc Natl Acad Sci USA, 2009, 106: 17255

2 Yarkony D R, Silbey R J. Variational approach to exciton transport in molecular crystals. J Chem Phys, 1977, 67: 5818

3 Cheng Y C, Silbey R J. A unified theory for charge-carrier transport in organic crystals. J Chem Phys, 2008, 128: 114713

4 Davids P S, Campbell I H, Smith D L. Device model for single carrier organic diodes. J Appl Phys, 1997, 82: 6319

5 Bässler H. Charge transport in disordered organic photoconductors: A Monte Carlo simulation study. Phys Status Solidi B, 1993, 175: 15

6 Tully J C. Perspective: Nonadiabatic dynamics theory. J Chem Phys, 2012, 137: 22A301

7 Troisi A, Orlandi G. Charge-transport regime of crystalline organic semiconductors: Diffusion limited by thermal off-diagonal electronic disorder. Phys Rev Lett, 2006, 96: 086601

8 Ciuchi S, Fratini S, Mayou D. Transient localization in crystalline organic semiconductors. Phys Rev B, 83: 081202

9 Tamura H, Tsukada M, Ishii $\mathrm{H}$, et al. Roles of intramolecular and 
intermolecular electron-phonon coupling on the formation and transport of large polarons in organic semiconductors. Phys Rev B, 2012, 86: 035208

10 Hannewald K, Stojanović V M, Schellekens J M T, et al. Theory of polaron bandwidth narrowing in organic molecular crystals. Phys Rev B, 2004, 69: 075211

11 Hains A W, Liang Z, Woodhouse M A, et al. Molecular semiconductors in organic photovoltaic cells. Chem Rev, 2010, 110: 6689

12 Coropceanu V, Cornil J, da Silva Filho D A, et al. Charge transport in organic semiconductors. Chem Rev, 2007, 107: 926

13 Konezny S J, Bussac M N, Zuppiroli L. Hopping and trapping mechanisms in organic field-effect transistors. Phys Rev B, 2010, 81: 045313

14 Zurek W H. Decoherence, einselection, and the quantum origins of the classical. Rev Mod Phys, 2003, 75: 715

15 Picon J D, Bussac M N, Zuppiroli L. Quantum coherence and carriers mobility in organic semiconductors. Phys Rev B, 2007, 75: 235106

16 Sakanoue T, Sirringhaus H. Band-like temperature dependence of mobility in a solution-processed organic semiconductor. Nat Mater, 2010, 9: 736

17 Pope M, Swenberg C E. Electronic Processes in Organic Crystals. Oxford: Oxford University Press, 1982

18 Wei S, Yao Y, Wu C Q. Theory of incoherent hopping transport with spin interactions in organic semiconductors. in press

19 Koster L J, Smits E C P, Mihailetchi V D, et al. Device model for the operation of polymer/fullerene bulk heterojunction solar cells. Phys Rev B, 2005, 72: 085205

20 Langevin P. Recombinaison et mobilites des ions dans les gaz. Ann Chim Phys, 1903, 28: 433

21 Koster L J, Mihailetchi V D, Blom P W M. Bimolecular recombination in polymer/fullerene bulk heterojunction solar cells. Appl Phys Lett, 2006, 88: 052104

22 Schockley W, Read W T. Statistics of the recombinations of holes and electrons. Phys Rev, 1952, 87: 835

23 Hall R N. Electron-hole recombination in germanium. Phys Rev, 1952, 87: 3878

24 Yao Y, Sun X Y, Ding B F, et al. A combined theoretical and experimental investigation on the transient photovoltage in organic photovoltaic cells. Appl Phys Lett, 2010, 96: 203306

25 Li D L, Si W, Yang W C, et al. Spike in transient photocurrent of organic solar cell: Exciton dissociation at interface. Phys Lett A, 2012, 376: 227

26 Yang W C, Li D L, Yao Y, et al. Enhanced surface losses of organic solar cells induced by efficient polaron pair dissociation at the metal/organic interface. J Appl Phys, 2012, 112: 034510

27 Yang W C, Yao Y, Wu C Q. Mechanisms of device degradation in organic solar cells: Influence of charge injection at the metal/organic contacts. Org Electron, 2013, 14: 1992

28 Pasveer W F, Cottaar J, Tanase C, et al. Unified description of charge-carrier mobilities in disordered semiconducting polymers. Phys Rev Lett, 2005, 94: 206601

29 Miller A, Abrahams E. Impurity conduction at low concentrations. Phys Rev, 1960, 120: 745

30 Marcus R A. On the theory of electron-transfer reactions. VI. Unified treatment for homogeneous and electrode reactions. J Chem Phys, 1965, 43: 679

31 Wetzelaer G A H, Koster L J A, Blom P W M. Validity of the einstein relation in disordered organic semiconductors. Phys Rev Lett, 2011, 107: 066605
32 Zhou J, Zhou Y C, Zhao J M, et al. Carrier density dependence of mobility in organic solids: A Monte Carlo simulation. Phys Rev B, 2007, 75: 153201

33 Meng L, Wang D, Li Q, et al. An improved dynamic Monte Carlo model coupled with Poisson equation to simulate the performance of organic photovoltaic devices. J Chem Phys, 2011, 134: 124102

34 Gershenson M E, Podzorov V, Morpurgo A F. Electronic transport in single-crystal organic transistors. Rev Mod Phys, 2006, 78: 973

35 Podzorov V, Menard E, Borissov A, et al. Intrinsic charge transport on the surface of organic semiconductors. Phys Rev Lett, 2004, 93: 086602

36 Matsui H, Mishchenko A S, Hasegawa T. Distribution of localized states from fine analysis of electron spin resonance spectra in organic transistors. Phys Rev Lett, 2010, 104: 056602

37 Devizis A, Meerholz K, Hertel D, et al. Ultrafast charge carrier mobility dynamics in poly(spirobifluorene-co-benzothiadiazole): Influence of temperature on initial transport. Phys Rev B, 2010, 82: 155204

38 Troisi A. Dynamic disorder in molecular semiconductors: Charge transport in two dimensions. J Chem Phys, 2011, 134: 034702

39 Li Y, Yi Y, Coropceanu V, et al. Symmetry effects on nonlocal electron-phonon coupling in organic semiconductors. Phys Rev B, 2012, 85: 245201

40 Böhlin J, Linares M, Stafström S. Effect of dynamic disorder on charge transport along a pentacene chain. Phys Rev B, 2011, 83: 085209

41 Ciuchi S, Fratini S. Band dispersion and electronic lifetimes in crystalline organic semiconductors. Phys Rev Lett, 106, 166403

42 Li Z Q, Podzorov V, Sai N, et al. Light quasiparticles dominate electronic transport in molecular crystal field-effect transistors. Phys Rev Lett, 2007, 99: 016403

43 Geng H, Peng Q, Wang L, et al. Toward quantitative prediction of charge mobility in organic semiconductors: Tunneling enabled hopping model. Adv Mater, 2012, 24: 3568

44 Wang L, Li Q, Shuai Z, et al. Multiscale study of charge mobility of organic semiconductor with dynamic disorders. Phys Chem Chem Phys, 2010, 12: 3309

45 Nan G, Yang X, Wang L, et al. Nuclear tunneling effects of charge transport in rubrene, tetracene, and pentacene. Phys Rev B, 2009, 79: 115203

46 Platt A D, Kendrick M J, Loth M, et al. Temperature dependence of exciton and charge carrier dynamics in organic thin films. Phys Rev B, 2011, 84: 235209

47 Landry B R, Subotnik J E. Standard surface hopping predicts incorrect scaling for Marcus' golden-rule rate: The decoherence problem cannot be ignored. J Chem Phys, 2011, 135: 191101

48 Landry B R, Subotnik J E. How to recover Marcus theory with fewest switches surface hopping: Add just a touch of decoherence. J Chem Phys, 2012, 137: 22A513

49 Flores J C. Iterative quantum local measurements and Anderson localization inhibition. Phys Rev B, 2004, 69: 012201

50 Yao Y, Si W, Hou X Y, et al. Monte Carlo simulation based on dynamic disorder model in organic semiconductors: From coherent to incoherent transport. J Chem Phys, 2012, 136: 234106

51 Heller E J. Frozen Gaussians: A very simple semiclassical approximation. J Chem Phys, 1981, 75: 2923

52 Kluk E, Herman M F, Davis H L. Comparison of the propagation of semiclassical frozen Gaussian wave functions with quantum propagation for a highly excited anharmonic oscillator. J Chem Phys, 1986, 84: 326

Open Access This article is distributed under the terms of the Creative Commons Attribution License which permits any use, distribution, and reproduction in any medium, provided the original author(s) and source are credited. 